

Figure 1 Keloid of the penis.

The penile keloid was treated by serial injections of intradermal triamcinolone under general anaesthetic. After four injections there was an appreciable reduction in both the size of the lesion and the itching.

\section{Discussion}

Keloid of the genitalia is exceedingly rare. It is intriguing that keloid has never been observed after

\section{References}

1. Brown, D. Hypospadias. Postgrad Med J 1949, 25: 367-375.

2. Crockett, D.J. Regional keloid susceptibility. Br J Plastic Surg 1964, 17: 245-253.

3. Parsons, R.W. A case of keloid of the penis. Plastic Reconstruct Surg 1966, 37: 431-432. the common procedure of circumcision. Denis Browne $^{1}$ stated that the skin of the penis 'never forms keloid' and Crockett ${ }^{2}$ found no example in a review of 250 Sudanese natives. In fact, two cases of penile keloid do exist in the literature. One case ${ }^{3}$ developed after surgical removal of a traumatic cyst on the shaft in an eight-year old Negro boy who, incidentally, had previously been circumcized without this complication. The second case ${ }^{4}$ followed a major laceration and subsequent burn to the shaft and scrotum of a 44 year old Caucasian.

Local pressure or irradiation are recognized methods of treating keloid ${ }^{5}$ but these techniques would be inappropriate in this anatomical area: it is impractical to apply firm and sustained compression, and irradiation is undesirable in such proximity to the testes. Excision is unreliable as recurrence and even exacerbation is likely to occur.

Local steroid injection is often effective in treating keloids, although its action is not fully understood. The triamcinolone is injected directly into the lesion in an amount sufficient to cause blanching; the injection can be repeated regularly although after a few doses there is a significant risk of local fat and skin atrophy.

4. Kormoczy, I. Enormous keloid (?) on the penis. Br J Plastic Surg 1978, 31: 268-269.

5. McGregor, I.A. Fundamental Techniques of Plastic Surgery, 8th ed. Churchill Livingstone, Edinburgh, 1989, pp. 18-22.

\title{
Resolution of danazol-induced cholestasis with S-adenosylmethionine
}

\author{
Gary P. Bray, J. Michael Tredger and Roger Williams
}

Institute of Liver Studies, King's College Hospital School of Medicine and Dentistry, Denmark Hill, London SE5 $8 R X, U K$

\begin{abstract}
Summary: In a case of danazol-induced cholestasis, the anti-cholestatic agent S-adenosylmethionine was given intravenously for 3 weeks and then orally for 6 weeks. This was well tolerated and led to prompt resolution of both jaundice and associated renal impairment.
\end{abstract}




\section{Introduction}

As with other androgens, there have been several reports of cholestasis induced by the $\mathrm{C} 17$ alkylated anabolic steroid danazol, ${ }^{1-4}$ a drug used in the treatment of disorders as varied as pelvic endometriosis, ${ }^{4}$ benign breast disease,${ }^{5}$ hereditary angioneurotic oedema ${ }^{6}$ idiopathic thrombocytopenia purpura $^{7}$ and haemolytic anaemia. Although liver function has returned to normal after withdrawal of danazol, this can take as long as 3 months even in the absence of jaundice. ${ }^{2}$ Treatment to accelerate normalization of liver function may therefore be of particular benefit in cases, such as the present one, in which severe symptoms occur.

\section{Case report}

The patient, a 60 year old male, had a 2 year history of chronic autoimmune haemolytic anaemia treated with prednisolone, azathioprine and splenectomy. In view of his refractory anaemia (haemoglobin $7.5 \mathrm{~g} / \mathrm{dl}$ ), he was started on danazol $200 \mathrm{mg}$ three times daily in April 1991, prior to which serum bilirubin was $42 \mu \mathrm{mol} / 1$ (normal less than 17), $\gamma$-glutamyl transpeptidase (GGT) was $80 \mathrm{IU} / 1$ (normal 12-62) and both alkaline phosphatase (ALP) and aspartate aminotransferase (AST) were normal. Cholecystectomy had been performed 2 years earlier and he had been taking warfarin for one year following a left leg deep venous thrombosis and multiple pulmonary emboli. He was also taking ranitidine, folic acid, phenoxymethylpenicillin and diclofenac.

Thirty-two days later, he complained of tiredness, nausea, left upper quadrant abdominal discomfort, pruritus, dark urine, pale stools and deep jaundice. Prothrombin time was greater than 150 seconds. Danazol and warfarin were discontinued and he was referred to the Institute of Liver Studies.

On examination, he had marked hepatomegaly with no clinical signs of chronic liver disease. Serum bilirubin rose from $326 \mu \mathrm{mol} / 1$ ( $85 \%$ conjugated) on initial admission to hospital to $666 \mu \mathrm{M}$ by the time of transfer and subsequently to a peak of $936 \mu \mathrm{mol} / \mathrm{l}$. Serum ALP rose to $336 \mathrm{IU} / \mathrm{l}$, GGT to $183 \mathrm{IU} / 1$ and AST to $120 \mathrm{IU} / 1$; serum albumin fell to a nadir of $33 \mathrm{~g} / \mathrm{dl}$. Serology for hepatitis A and $B$, auto-antibody screen and $\alpha$-fetoprotein were negative. Serum IgG was normal $(7.2 \mathrm{~g} / \mathrm{l})$ but both IgA and IgM were low $-0.52 \mathrm{~g} / 1$ (normal $1.2-4.5$ ) and $0.36 \mathrm{~g} / 1$ (normal $0.47-2.2$ ), respectively. Ultrasound scanning revealed that the liver was of normal reflectivity. Histological examination of his liver biopsy taken 24 days after stopping danazol showed features of drug-induced cholestasis with mild cholangiolytic changes and focal perivenular cell loss. Despite a slight reduction in the serum bilirubin after discontinuation of danazol, he remained deeply jaundiced with a fluctuating serum bilirubin of up to $733 \mu \mathrm{mol} / \mathrm{l}$ and serum creatinine rising to $227 \mu \mathrm{mol} / 1$ (Figure 1).

S-Adenosylmethionine (SAMe) ('Samyr'; Bioresearch, Milan, Italy) by intravenous infusion was commenced at a dose of $800 \mathrm{mg}$ twice daily for 10 days reducing to $800 \mathrm{mg}$ daily for a further 10 days. This resulted in a prompt decrease in the level of jaundice from a serum bilirubin of $476-44 \mu \mathrm{mol} / 1$ over 7 weeks and an improvement in renal function (Figure 1) and symptoms. Once the serum bilirubin had fallen to $137 \mu \mathrm{mol} / 1$ and the serum creatinine to within normal limits $(84 \mu \mathrm{mol} / \mathrm{l})$, the patient was discharged home on oral SAMe $(2,400 \mathrm{mg}$ daily). This was continued for a further 6 weeks. At this time, his serum bilirubin had returned to that observed prior to danazol $(44 \mu \mathrm{mol} / \mathrm{l})$ and he was symptomatically well.

\section{Discussion}

Although transient mild elevations of serum AST, ALP or both may occur in up to $14 \%$ of patients who take danazol, ${ }^{6}$ severe reactions seem to be rare. Over the 10 year period from 1981 to 1991,25 cases of hepatocellular impairment in patients taking danazol were reported to the Committee on Safety of Medicines (personal communication) but no deaths were recorded. To date only one death has been reported due to the hepatic effects of danazol, although non-A non-B hepatitis could not be excluded as the cause of the fulminant hepatic failure and aplastic anaemia in that case. ${ }^{8}$ For both clinical and subclinical cases of danazol-induced cholestasis, resolution after drug withdrawal is often slow. ${ }^{9}$ Our patient had particularly deep jaundice (probably exacerbated by his chronic

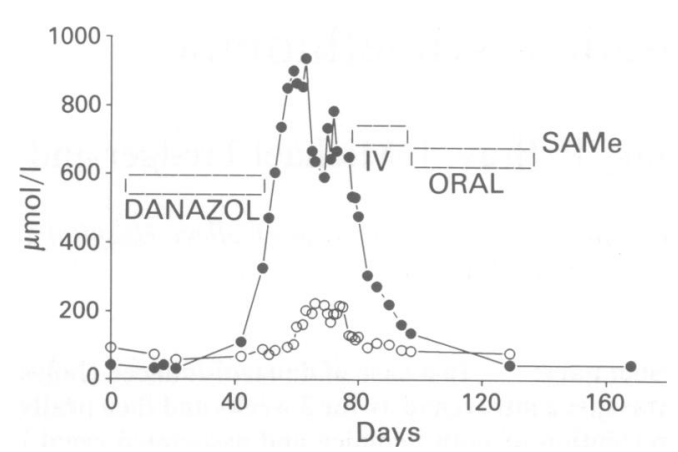

Figure 1 Relationship of danazol and S-adenosylmethionine (SAMe) treatment to serum bilirubin (normal $<17 \mu \mathrm{mol} / \mathrm{l}$ ) $(O)$ and creatinine (normal $<120 \mu \mathrm{mol} / \mathrm{l}$ ) (O). 
haemolytic state) together with renal impairment. The histological features in our patient were characteristic of androgen-induced cholestasis ${ }^{9}$ rather than a reaction to diclofenac, which is usually associated with more hepatitic features. ${ }^{10}$

The cholestatic effects of androgens and oestrogens are similar with their potential to induce cholestasis being determined by alkylation at the C17 alpha position. At a cellular level, these compounds act principally by impairing membrane fluidity which leads to a decrease in $\mathrm{Na}^{+} / \mathrm{K}^{+}$ ATPase activity. The model 17-alkylated steroid, ethinyl oestradiol, reduces sinusoidal uptake of bile acids and other organic anions, as well as the ability of hepatocytes to secrete taurocholate and bilirubin into the biliary canaliculus. ${ }^{9,11}$

The anticholestatic effects of SAMe are poorly understood, although the stable salt of this metabolite has been used successfully in cholestasis due to many causes (including pregnancy and chronic liver disease). As it is the principal methyl donor in man, SAMe may act by promoting methylation of phospholipid leading to an increase in plasma membrane content of phosphatidylcholine and an improvement in membrane fluidity. ${ }^{12}$ In animal models of oestrogen-induced cholestasis, SAMe has been shown to partially reverse the depression of bile flow and inhibition of $\mathrm{Na}^{+} / \mathrm{K}^{+}$ATPase activity caused by ethinyl oestradiol. SAMe may also reduce oestrogen-induced

\section{References}

1. Pearson, K. \& Zimmerman, H.J. Danazol and liver damage. Lancet 1988 , i: $645-646$.

2. Boue, F., Coffin, B. \& Delfraissy, J.-F. Danazol and cholestatic hepatitis. Ann Intern Med 1986, 105: 139-140.

3. Silva, M.O., Reddy, K.R., McDonald, T., Jeffers, L.J. \& Schiff, E.R. Danazol-induced cholestasis. Am J Gastroenterol 1989, 84: 426-428.

4. Xiol, X., Martina-Lacasa, J., Pons, M., Marti, E. \& Casais, L. Jaundice after danazol therapy for endometriosis. Am J Gastroenterol 1989, 84: 834-835.

5. Asch, R.H. \& Greenblatt, R.B. The use of an impeded androgen-danazol-in the management of benign breast disorders. Am J Obstet Gynecol 1977, 127: 130-134.

6. Hosea, S.W., Santaella, M.L., Brown, E.J., Berger, M. Katusha, K. \& Frank, M.M. Long-term therapy of hereditary angioedema with danazol. Ann Intern Med 1980, 93: 809-812.

7. Ahn, Y.S., Harrington, W.J., Simon, S.R., Mylvaganam, R., Pall, L.M. \& So, A.G. Danazol for the treatment of idiopathic thrombocytopenic purpura. N Engl J Med 1983, 308, 1396-1399.

8. Nakajima, T., Mizushima, N., Matsuda, H. et al. Fulminant hepatic failure associated with aplastic anaemia after treatment with danazol. Case report. Br J Obstet Gynaecol 1986, 93: $1013-1015$ cholestasis by promoting cysteine synthesis and increasing sulphation of bile acids, thereby decreasing the proportion of the cholestatic glucuronide conjugates. $^{12}$ Alternatively SAMe may stimulate hepatic glutathione (GSH) synthesis ${ }^{13}$ leading to a rise in activity of GSH-dependent enzymes (such as GSH transferase) and intracellular ATP ${ }^{14}$ and an amelioration in cholestasis.

In view of these findings in animal models of cholestasis, SAMe may be particularly effective in sex-hormone-related cholestasis. ${ }^{12}$ Although the use of SAMe has been described to improve abnormalities of liver function tests associated with the oral contraceptive pill, ${ }^{15}$ there is only one report, published in full, of its use in human drug-induced cholestasis. Although the drug involved in that particular case is not documented, administration of SAMe led to normalization of serum bilirubin. ${ }^{16}$

As treatment with SAMe is well tolerated, it should be considered in patients with severe druginduced cholestatis, particularly those in whom it is related to the administration of androgens or oestrogens.

\section{Acknowledgement}

We would like to thank Dr Bernard Portmann for review of liver histology.
9. King, P.D. \& Blitzer, L. Drug-induced cholestasis: pathogenesis and clinical features. Semin Liver Dis 1990, 10: $316-321$.

10. Iveson, T.J., Ryley, N.G., Kelly, P.M.A. et al. Diclofenac associated hepatitis. $J$ Hepatol 1990, 10: 85-89.

11. Meier-Abt, P.J. Cellular mechanisms of intrahepatic cholestasis. Drugs 1990, 40 (Suppl 3): 84-97.

12. Almasio, P., Bortoloini, M., Pagliaro, L.\& Coltorti, M. Role of S-adenosyl-L-methionine in the treatment of intrahepatic cholestasis. Drugs 1990, 40 (Suppl 3): 111-123.

13. Bray, G.P., Tredger, J.M. \& Williams, R. S-Adenosylmethionine protects against acetaminophen hepatotoxicity in two mouse models. Hepatology 1992, 15: 297-301.

14. Montero, C., Smolenski, R.T., Duley, J.A. \& Simmonds, H.A. S-Adenosylmethionine increases erythrocyte ATP in vitro by a route independent of adenosine kinase. Biochem Pharmacol 1990, 40: 2617-2623.

15. Frezza, M., Pozzato, G., Pison, G., Zalateo, C., Chiesa, L. \& Di Padova, C. S-Adenosylmethione counteracts oral contraceptive hepatotoxicity in women. Am J Med Sci 1987, 293: 234-238.

16. Adachi, Y., Nanno, T., Kanbe, A. et al. The effects of S-adenosylmethionine on intrahepatic cholestasis. Jap Arch Intern Med 1986, 33: 185-192. 